\title{
2035 Immunohistological basis for late gadolinium enhancement phenomenon in tako tsubo cardiomyopathy on cardiovascular magnetic resonance imaging
}

\author{
Andreas Rolf*1, Holger Nef1 ${ }^{1}$, Helge Moellmann11, Sawa Kostin², \\ Christian Troidl2 ${ }^{2}$, Sandra Voss ${ }^{2}$, Johannes Rixe ${ }^{1}$, Guido Conradi1, \\ Michael Weber ${ }^{1}$, Albrecht Elsaesser ${ }^{1}$, Christian Hamm ${ }^{1}$ and Thorsten Dill ${ }^{1}$
}

Address: ${ }^{1}$ Kerckhoff-Heart-Center, Bad Nauheim, Germany and ${ }^{2}$ Max-Planck-Institute for Heart and Lung Research, Bad Nauheim, Germany

* Corresponding author

from I th Annual SCMR Scientific Sessions

Los Angeles, CA, USA. I-3 February 2008

Published: 22 October 2008

Journal of Cardiovascular Magnetic Resonance 2008, I0(Suppl I):A304 doi:10.I I86/I532-429X-I0-SI-A304

This abstract is available from: http://jcmr-online.com/content//0/SI/A304

(c) 2008 Rolf et al; licensee BioMed Central Ltd.

\section{Objective}

The absence of Late Gadolinium Enhancement (LGE) in patients with Tako Tsubo Cardiomyopathy (TTC) has been a widely accepted paradigm. Recently several authors reported single cases of LGE, the reason for this phenomenon yet unknown. LGE can be attributed to one or more of the following tissue conditions: edema, necrosis or fibrosis. As published previously we found transient interstitial fibrosis in myocardial biopsies of TTC patients. We therefore sought to determine, if the LGE phenomenon in TTC is based on a transient increase in extracellular fibroid matrix.

\section{Methods}

The diagnosis of TTC was based on apical ballooning with hyper contractile basal segments after rule out of CHD on $\mathrm{X}$-ray coronary angiograms. Written informed consent was obtained from all patients to undergo biopsy. Viral myocarditis was ruled out by PCR. We performed collagen-1 staining on the myocardial biopsies and computed the mean percentage areas of collagen per field of view. Creatininkinase levels were compared as markers of necrosis. Patients underwent cardiac MRI (CMR). Late Enhancement was documented on inversion recovery Fast Gradient Echo Sequences (FLASH, Siemens) and graded as present or absent. Edema was calculated as mean percentage area of the myocardium on Turbo Spin Echo
Sequences in two long axis orientations. Mann-Whitney$\mathrm{U}$-Test were used to compare group differences. Absolute values are given \pm SD. P-values equal or less than 0.05 were considered significant.

\section{Results}

Complete data were available for 8 patients, two of which were LGE positive. There was no significant difference in CK values or edema between groups (CK: LGE pos. 115.5 $\pm 48.8 \mathrm{U} / \mathrm{L}$ vs. LGE neg. $138.6 \mathrm{U} / 1 \mathrm{p}=0.7$; edema: LGE pos. $18.7 \pm 0.2 \%$ vs. LGE neg. $21.3 \pm 15.8 \% \mathrm{p}=0.56$ ). The extracellular matrix however, as represented by collagen-1 staining, was significantly increased in LGE positive Patients (LGE pos. $12 \pm 4.8 \%$ vs. LGE neg. $4.6 \pm 1.8 \%$ p = $0.046)$.

\section{Conclusion}

The present data suggest that LGE in patients with TTC is caused by transient increase of extracellular matrix, contradicting the paradigm, that LGE rules out TTC. A limitation of the study is the small sample size due to the low frequency of the disease. Therefore further studies with larger patient cohorts are warranted to corroborate these findings. 